\title{
The Development of Texture in Co-Cr Films
}

\author{
Li Cheng-Zhang, J. C. Lodder, and J. A. Szpunar
}

\begin{abstract}
Texture development as a function of film thickness $(5-980 \mathrm{~nm})$ was investigated for two series of $\mathrm{Co}_{81} \mathrm{Cr}_{19}$ films. In general, the films were strongly textured. The orientation ratio, $O R_{c}$, was used to describe the strength of the texture. Experimental data showed that for the Series A films (5$200 \mathrm{~nm}$ ), the $O R_{c}$ value increased with increasing film thickness, while for Series B films $(46-980 \mathrm{~nm})$, the $O R_{c}$ as a function of the film thickness described a single peak curve, with its maximum near $130 \mathrm{~nm}$. The calculated local orientation ratios $O R_{c x}$ for both the Series $A$ and $B$ films had maxima near 110 $\mathrm{nm}$. The strain in $\mathrm{Co}-\mathrm{Cr}$ films also changes with the film thickness. In the case of the Series B films, the strain along the film normal gradually changed from a tensile to a compressive strain with increasing film thickness, and near $130 \mathrm{~nm}$ the film was in a stress-free condition. It was also discovered that for $\mathrm{Co}-\mathrm{Cr}$ films thinner than $46 \mathrm{~nm}$, the aspect ratio of the grains approaches 1 and the typical columnar structure of grains is not observed, although (0002) fibre texture still exists.
\end{abstract}

\section{INTRODUCTION}

$\mathbf{I}^{\mathrm{T}}$ is important to understand the nucleation mechanism and the process of grain growth in thin films as the developed microstructure will control the resulting magnetic properties. At present, the magnetic thin films used in both longitudinal and perpendicular recording media are polycrystalline and have a thickness in the range of 15-240 $\mathrm{nm}$ [1], [2], and recent increases in the recording density of magnetic disks pushes this range to even thinner films. The magnetic and mechanical properties of these thin films can vary, depending on the condition of the film-deposition process, the composition, the microstructure, the grain morphology and the texture.

Previous investigations [6], [9]-[12] have demonstrated that $\mathrm{Co}-\mathrm{Cr}$ films display a columnar microstructure with a strong degree of alignment of the $\mathrm{C}$-axis in the direction perpendicular to the film normal. The high degree of texture in $\mathrm{Co}-\mathrm{Cr}$ films plays a major role in optimizing their magnetic properties.

There is clear experimental evidence [5]- [7] that Co$\mathrm{Cr}$ film texture is closely related to the choice of the substrate and/or seedlayer. In general, the dispersion of the orientation of the hcp $\mathrm{C}$-axis formed by the "layer-is-

Manuscript received August 19, 1992; revised September 21, 1993.

L. Cheng Zeng is on leave from Institute of Computing Technology, Academia Sinica, P.O. Box 2704-6, Beijing, P.R. China 100080. He is currently with Department Metallurgical Eng., McGill University, 3450 University Street, Montreal, PQ, Canada, H3A 2A7

J. C. Lodder is with MESA Research Institute, Faculty of Electrical Eng., University of Twente, P.O. Box 217,7500 AE Enschede, The Netherlands.

J. A. Szpunar is with Department Metallurgical Eng., McGill University, 3450 University Street, Montreal, PQ, Canada, H3A 2 A7.

IEEE Log Number 9400798. land" growth mechanism is smaller than that for the "island" growth mechanism. It has been shown [6] that for specimens with a $\mathrm{Co}-\mathrm{Cr} / \mathrm{Si}$ structure, the growth of the columnar grains can be described by the "layer-island" growth mechanism.

Texture develops as a result of competition between nuclei and grains having different orientation. In order to describe the growth mechanism, the textural evolution and changes in strain were studied for five Series of $\mathrm{Co}-\mathrm{Cr}$ films. Our research concentrated on the investigation of the following:

a) The textural changes as a function of the film thickness for $\mathrm{Co}-\mathrm{Cr}$ films having the same average composition.

b) The textural changes in films having different compositions, but having the same thickness.

c) The influence of the substrate and/or seedlayer on the texture of Co-Cr films with the same thickness.

d) The influence of different preparation methods (i.e., both sputtering and two-beam obliquely co-evaporation methods) on the textural evolution.

e) The correlation between textural development, strain distribution and magnetic properties in $\mathrm{Co}-$ Cr films.

In this report, we describe the textural evolution with the thickness for $\mathrm{Co}_{81} \mathrm{Cr}_{19}$ films. Research on other topics will be reported in other papers [10], [12].

\section{Experimental Procedure}

Two series of $\mathrm{Co}_{81} \mathrm{Cr}_{19}$ films, obtained by sputtering, were chosen to investigate textural evolution and the change in strain with film thickness. The average nominal composition for all tested specimens was $\mathrm{Co}_{81} \mathrm{Cr}_{19}$. The preparation conditions were as follows: $P_{a r}$ (Argon pressure) $=4 \times 10^{-3} \mathrm{mbar}$, voltage, $V_{r f}=1.6 \mathrm{kV}$ and $T_{s}$ (substrate temperature) $=150^{\circ} \mathrm{C}$. The Series $\mathrm{A}$ and $\mathrm{B}$ films were deposited on $\mathrm{Ge} / \mathrm{SiO}_{2} / \mathrm{Si}$ and $\mathrm{Si}$ substrates. The thickness of the films and the surface roughness were measured using STM (scanning tunneling microscope) and AFM (atomic force microscope) methods. The thicknesses for Series A and B are in the range of 5-200 nm and 46-980 $\mathrm{nm}$, respectively. The maximum surface roughness for the thinnest films is about $6 \mathrm{~nm}$ and increases with increasing film thickness up to about $50 \mathrm{~nm}$. The magnetic properties were measured by a VSM and a torque magnetometer. The maximum applied field was about $840 \mathrm{kA} / \mathrm{m}$. The coercivities $H_{c}$ for all tested films 
TABLE I

Properties for Series A $\mathrm{Co}_{81} \mathrm{Cr}_{19}$ FILMS

\begin{tabular}{lcccccc}
\hline No & $\begin{array}{c}\text { Thickness } \\
(\mathrm{nm})\end{array}$ & $\begin{array}{c}M_{s} \\
\mathrm{kA} / \mathrm{m}\end{array}$ & $\begin{array}{c}H_{\mathrm{c} \perp} \\
\mathrm{kA} / \mathrm{m}\end{array}$ & $\begin{array}{c}H_{\mathrm{c} \|} \\
\mathrm{kA} / \mathrm{m}\end{array}$ & $\begin{array}{c}K_{1} \\
\mathrm{~kJ} / \mathrm{m}^{3}\end{array}$ & $R_{s \perp}$ \\
\hline $\mathrm{A}_{1}$ & 5 & 360 & 4.0 & 3.1 & 122 & 1.0 \\
$\mathrm{~A}_{2}$ & 12 & 460 & 4.3 & 1.7 & 116 & 0.02 \\
$\mathrm{~A}_{3}$ & 25 & 500 & 15.4 & 16.8 & 127 & 0.06 \\
$\mathrm{~A}_{4}$ & 50 & 480 & 36.8 & 12.9 & 110 & 0.11 \\
$\mathrm{~A}_{5}$ & 100 & 510 & 47.2 & 11.8 & 134 & 0.12 \\
$\mathrm{~A}_{6}$ & 200 & 500 & 69.9 & 12 & 131 & 0.17 \\
\hline
\end{tabular}

TABLE II

Properties for SERIEs B $\mathrm{Co}_{81} \mathrm{Cr}_{19}$ Films

\begin{tabular}{lccccrc}
\hline No & $\begin{array}{c}\text { Thickness } \\
(\mathrm{nm})\end{array}$ & $\begin{array}{c}M_{s} \\
\mathrm{kA} / \mathrm{m}\end{array}$ & $\begin{array}{c}H_{\mathrm{c} \perp} \\
\mathrm{kA} / \mathrm{m}\end{array}$ & $\begin{array}{c}H_{\mathrm{c} \perp} \\
\mathrm{kA} / \mathrm{m}\end{array}$ & $\begin{array}{r}K_{1} \\
\mathrm{~kJ} / \mathrm{m}^{3}\end{array}$ & $R_{\text {s }}$ \\
\hline $\mathrm{B}_{1}$ & 46 & 450 & 79 & 7 & 94 & 0.26 \\
$\mathrm{~B}_{2}$ & 110 & 470 & 96 & 6 & 115 & 0.25 \\
$\mathrm{~B}_{3}$ & 300 & 445 & 65 & 6 & 99 & 0.16 \\
$\mathrm{~B}_{4}$ & 610 & 435 & 46 & 7 & 84 & 0.11 \\
$\mathrm{~B}_{5}$ & 980 & 430 & 40 & 7 & 86 & 0.10 \\
\hline
\end{tabular}

range from 4 to $96 \mathrm{kA} / \mathrm{m}$. The most relevant properties of the two Series of $\mathrm{Co}_{81} \mathrm{Cr}_{19}$ films are presented in Tables 1 and 2. $R_{s \perp}$ is the squareness ratio $\left(M_{r \perp} / M_{s}\right)$ in the direction normal to the film plane. $H_{c \perp}$ and $H_{c \|}$ are the coercivities in the direction normal to the film and the in-plane direction, respectively.

To investigate the crystallographic texture, all specimens were first measured using a Rigaku diffractomer $\left(\mathrm{Cu}-\mathrm{K}_{\alpha}\right.$ radiation) to determine the crystalline structure. The (0002) pole figure of the same specimens were then measured using a Siemens D-500 texture goniometer ( $\mathrm{Mo}-$ $K_{\alpha}$ radiation). The measured pole figures show a strong maxima in the central part, and this range was then remeasured over $12^{\circ}$ angular intervals as a function of the specimen tilt angle $\alpha$ (i.e., the radial angle of the pole figure). The ratio $O R_{c}(\alpha, \beta)$ of the pole density $F(\alpha, \beta)$ of the film to the pole density of a random specimen was used to evaluate the orientation ratio using the following expression:

$$
O R_{c}(\alpha, \beta)=\frac{\left(\frac{1}{\alpha_{1}-\alpha_{2}}\right) \int_{\alpha_{1}}^{\alpha_{1}} \int_{0}^{2 \pi} F(\alpha, \beta) d \alpha d \beta}{\frac{2}{\pi} \int_{0}^{\pi / 2} \int_{0}^{2 \pi} F(\alpha, \beta) d \alpha d \beta},
$$

where $\beta$ is the circumferential angle of the pole figure. In our case, the value of $F(\alpha, \beta)$ is assumed to be equal to the value of $F(\alpha)$, since the texture in the $\alpha$-Co phase exhibits high degree of rotational symmetry around the film normal. For the sake of convenience, the maximum of the $O R_{c}(\alpha, \beta)$ value defined in (1) will be called the orientation ratio $O R_{c}$ of the crystallites for the $(0002)$ plane.

\section{Results AND Discussion}

The measured relation between the columnar diameter and thickness for Series B films is illustrated in Fig. 1.

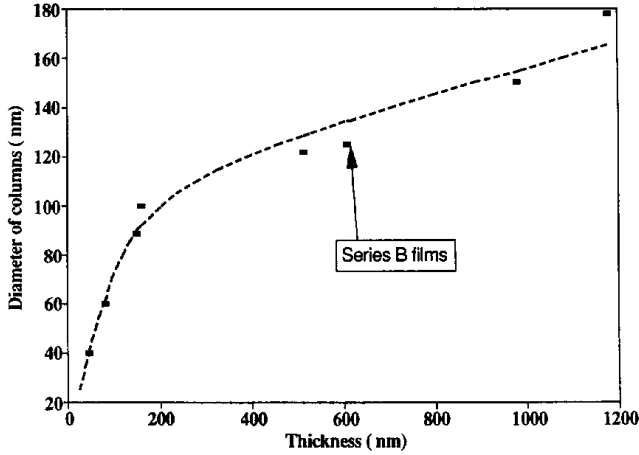

Fig. 1. The dependence of the columnar diameter on the thickness for Series $\mathrm{B} \mathrm{Co}_{81} \mathrm{Cr}_{19}$ films.

As expected, the diameter of the columnar grain increases with increasing film thickness in the range of $46-980 \mathrm{~nm}$, but the rate of increase of this diameter for films thinner than $200 \mathrm{~nm}$ is much higher than that in films thicker than this. Thus during the initial stages of grain growth, the grains grow fastest in the direction parallel to the film surface but this growth rate gradually decreases and approaches a saturation value for film thicknesses greater than $500 \mathrm{~nm}$. Similar results were also reported in reference [15].

Two columnar growth mechanisms have been observed. In the first case cross-sectional TEM and SEMmicrographs showed [6], [11] that the columns in sputtered $\mathrm{Co}-\mathrm{Cr}$ films stretch straight upwards and have a near constant diameter along the columnar length. Other SEM investigations [15] have shown that the columns increase slightly in diameter towards the film surface from bottom to top. The grains in our specimens belonged to this category of conically shaped columns. Since the columnar grains were observed growing throughout the thickness of all specimens it is reasonable to assume that the length of a column in a $\mathrm{Co}-\mathrm{Cr}$ film is equal to the film thickness. Based on this assumption the ratio T/D of the grain length $T$ to diameter D, the "aspect ratio," was calculated. As shown in Fig. 2, the aspect ratio as a function of the film thickness for the Series B films exhibited a linear relationship for films up to about $1000 \mathrm{~nm}$. As the thickness decreased to $40 \mathrm{~nm}$, the aspect ratio approaches 1 and the grains tend to form an equiaxed crystallite structure.

Diffraction spectra were used to identify crystallographic phases in the films. Typical diffraction spectra for specimens $A_{5}(100 \mathrm{~nm})$ and $A_{1}(5 \mathrm{~nm})$ are shown in Figs. 3 and 4(a), respectively. To distinguish between the diffraction spectra of the Co-Cr film and the Si substrate, the diffraction spectrum for the $\mathrm{Si}$ substrate is shown in Fig. 4(b). It can be observed from these diffraction patterns that the (0002) and (0004) maxima for hcp $\alpha$-Co structures are identified. The (200) and (400) maxima of the cubic structure of the Si substrate can also be identified. Furthermore, the FCC diffraction maxima for the $\mathrm{CoO}$ phase can be identified by the presence of (220) planes. 


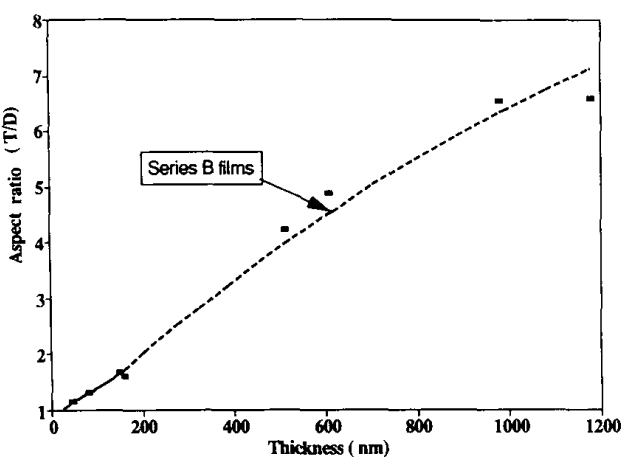

Fig. 2. The dependence of the aspect ratio on the thickness for Series B $\mathrm{Co}_{81} \mathrm{Cr}_{19}$ films.

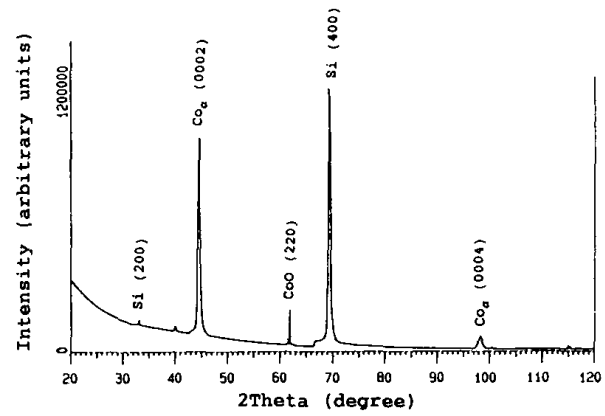

Fig. 3. The diffraction spectrum for specimen $A_{5}(100 \mathrm{~nm})$.

Varying the film thickness shifts the peak (0002). For both series a systematic increase in the (0002) peak position is observed with increasing film thickness, as is shown by experimental data in Tables III and IV.

Two possible effects could influence this (0002) peak position shift. The first [14] is the existence of an FCC Co phase in the $\mathrm{Co}_{81} \mathrm{Cr}_{19}$ films. In our case, however, the measured diffraction spectra can not furnish any evidence to support this case. The other explanation for the peak shift is the existence of internal stresses within the films. When a $\mathrm{Co}-\mathrm{Cr}$ film is subjected to a tensile or compressive stress, the lattice plane spacing in the grains will change from their stress-free value to a new value corresponding to the magnitude and sign of the applied stress. This macrostrain leads to a shift of the diffraction lines to new $2 \theta$ positions. Internal stress in a $\mathrm{Co}-\mathrm{Cr}$ film is usually closely related to the type of the substrate and/or the seedlayer.

Based on the peak-shift data listed in Tables III and IV, and diffraction pattern of annealed powders, the strain in the films can be calculated. The calculated strain $\epsilon$ is plotted as a function of film thickness in Fig. 5. For Series B films, as the thickness increases, the sign of the strain $\epsilon$ along the film normal gradually changes from positive to negative. Thus the thinner films are subjected to a tensile strain, and films thicker than $130 \mathrm{~nm}$ are under a compressive strain. In the vicinity of $130 \mathrm{~nm}$, then, the film is in a near stress-free condition. For Series A films, the

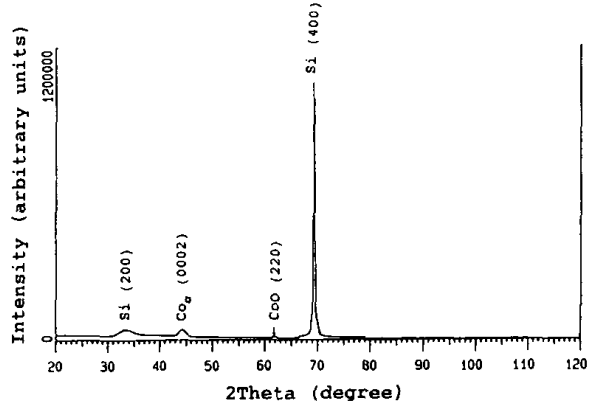

(a)

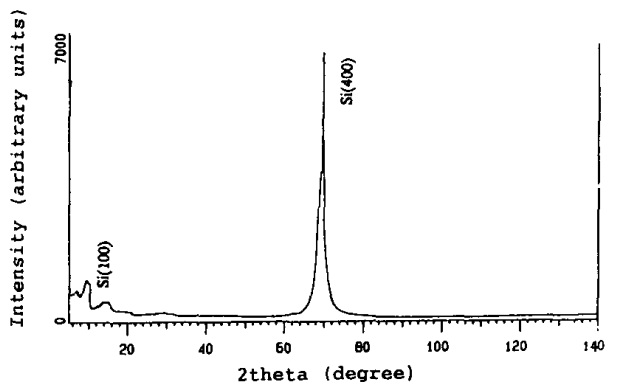

(b)

Fig. 4. (a) The diffraction spectrum for specimen $A_{1}(5 \mathrm{~nm})$. (b) The diffraction spectrum for $\mathrm{Si}$ substrates.

strain decreases monotonically with increasing thickness, and in the vicinity of $200 \mathrm{~nm}$ the strain is almost equal to zero. It can be concluded from these results that the magnitude and sign of the strain in $\mathrm{Co}-\mathrm{Cr}$ films are strongly influenced by the film thickness.

There are a number of possible explanations as to the origin of stress in the films. One is a perpendicular surface relaxation [17], [19] which results from a charge redistribution at the film surface due to the lowered symmetry of the surface electronic structure. In our case, the surface layer seems to experience a biaxial tensile strain when compared to the spatial distribution of the strain in the interior of the film. This observation is supported by the surface layer strain versus thickness curve plotted in Fig. 5 . Here, the surface strain $c_{s}$ was calculated by analysing the peak shift of the FCC (220) CoO phase. Auger depth profiling has proven [20]-[21] that at the outer surface (= 6A) of a Co-Cr film only the $\mathrm{CoO}$ and $\mathrm{CoCr}$ phases are present, while the inner oxide mainly consists of $\mathrm{Cr}_{2} \mathrm{O}_{3}$.

Another explanation for the observed film stress may be due to planar lattice misfits which may originate from a mismatch of the lattice spacing in the interfacial layer between the $\mathrm{Co}-\mathrm{Cr}$ film and the Si substrate, as well as the Ge seedlayer if present. One last possible explanation may be that the planar film strain is caused by varying thermal expansion coefficients between the $\mathrm{Co}-\mathrm{Cr}$ film and the substrate.

At present, there is no strong experimental evidence 
TABLE III

The Dependence of the Peak Shift of (0002) Planes on the Thickness for Series A

\begin{tabular}{lcccccc}
\hline \multicolumn{1}{c}{ No } & $\mathrm{A}_{1}$ & $\mathrm{~A}_{2}$ & $\mathrm{~A}_{3}$ & $\mathrm{~A}_{4}$ & $\mathrm{~A}_{5}$ & $\mathrm{~A}_{6}$ \\
\hline Thickness (nm) & 5 & 12 & 25 & 50 & 100 & 200 \\
$2 \theta$ (degree) & $44.12^{\circ}$ & $44.35^{\circ}$ & $44.36^{\circ}$ & $44.45^{\circ}$ & $44.46^{\circ}$ & $44.49^{\circ}$ \\
\hline
\end{tabular}

TABLE IV

The Dependence of the Peak Shift of (0002) Planes on the Thickness for Series B

\begin{tabular}{cccccc}
\hline \multicolumn{1}{c}{ No } & B $_{1}$ & $\mathbf{B}_{2}$ & $\mathbf{B}_{3}$ & $\mathbf{B}_{4}$ & $\mathbf{B}_{5}$ \\
\hline Thickness (nm) & 46 & 110 & 300 & 610 & 980 \\
$2 \theta$ (degree) & $43.84^{\circ}$ & $44.49^{\circ}$ & $44.73^{\circ}$ & $44.84^{\circ}$ & $44.91^{\circ}$ \\
\hline
\end{tabular}

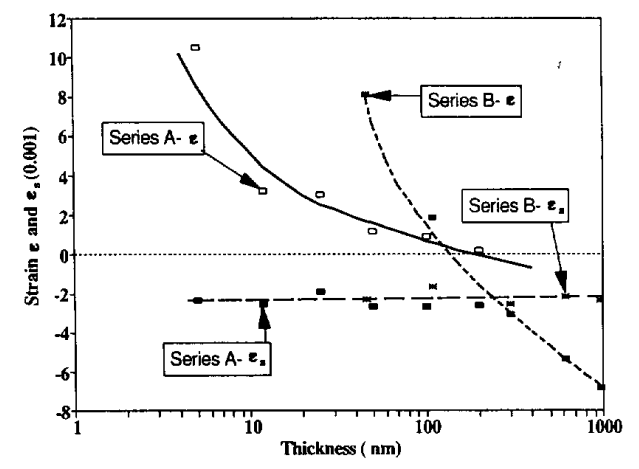

Fig. 5. The dependence of the strain of the whole film $\epsilon$ and the strain of surface layer $\epsilon_{s}$ on the thickness for Series A and B films.

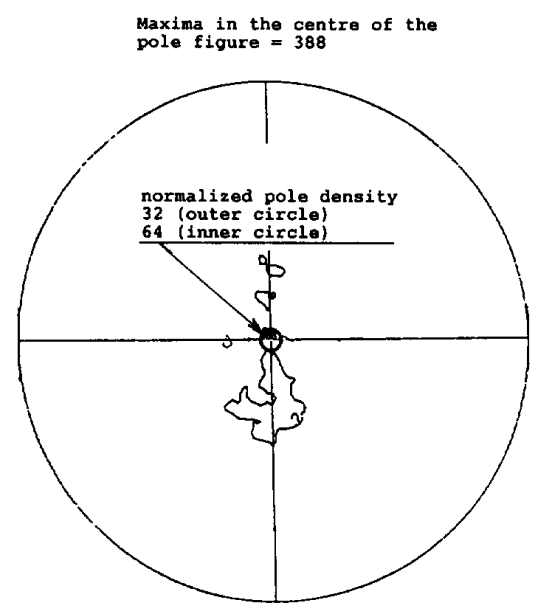

Fig. 6. The (0002) pole figure for specimen $A_{5}(100 \mathrm{~nm})$.

pointing out which one, or combination of, these factors exert a dominant influence on the distribution pattern of the strain in the investigation $\mathrm{Co}_{81} \mathrm{Cr}_{19}$ films. However, the measured changes in the strain and texture for specimens having different thickness are closely related.

Typical results of the texture measurements are presented in the form of (0002) pole figures in Figs. 6 and 7 for specimen $A_{5}(100 \mathrm{~nm})$ and $A_{2}(12 \mathrm{~nm})$. As shown in

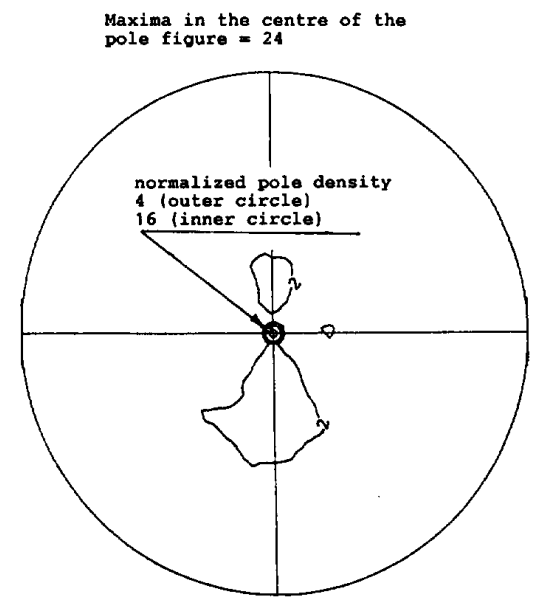

Fig. 7. The (0002) pole figure for specimen $A_{2}(12 \mathrm{~nm})$.

Fig. 6, the $100 \mathrm{~nm}$ film exhibits a very strong texture with a maximum as high as 388 appearing at the centre of the (0002) pole figure. The pole figure consists of a series of very closely packed equal density curves in the centre which indicates a high degree of rotational symmetry. This demonstrates that the $(0002)$ plane is aligned parallel to the specimen surface. Pole figures for the remainder of the Series A and B films have a very similar texture to that of the $100 \mathrm{~nm}$ thick film, the exception being that the strength of the texture varies.

To evaluate the magnitude of the orientation ratio and to determine the influence of texture development on the material magnetic properties, more precise distributions of the crystallographic orientation for all tested films were measured using the Siemens texture goniometer. Typical distribution curves of the (0002) poles in the vicinity of the film normal are shown in Figs. 8 and 9.

Using the measured pole density distribution and (1), the normalized orientation ratio $O R_{c}(\alpha)$ of the $(0002)$ crystallographic planes can be calculated in random units. The angular dependence of the orientation ratio $O R_{c}(\alpha)$ for Series A and B films is presented in Figs. 10 and 11. It can be seen from Fig. 10 that, as the thickness increases 


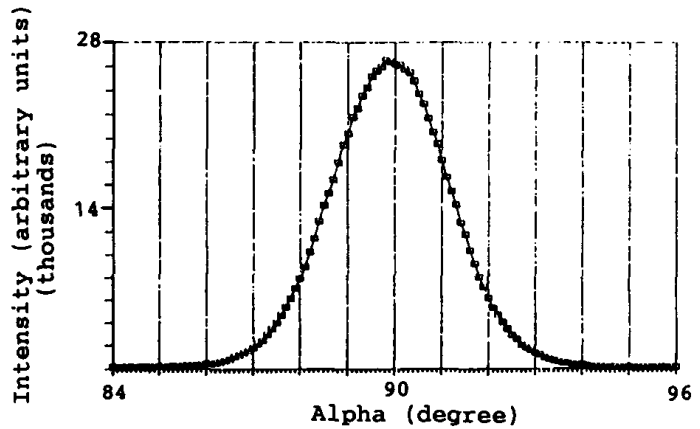

Fig. 8. (0002) pole density as a function of the specimen tilt angle $\alpha$ for specimen $A_{5}(100 \mathrm{~nm})$

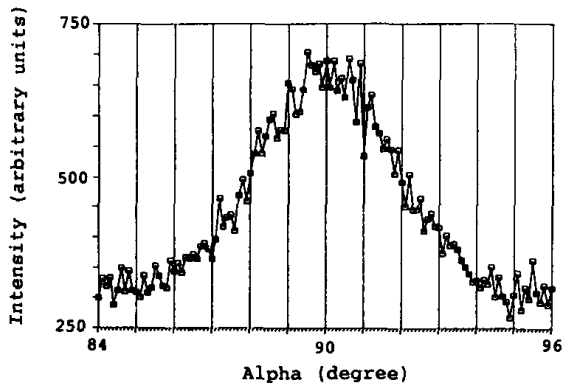

Fig. 9. (0002) pole density as a function of the specimen tilt angle $\alpha$ for specimen $A_{1}(5 \mathrm{~nm})$.

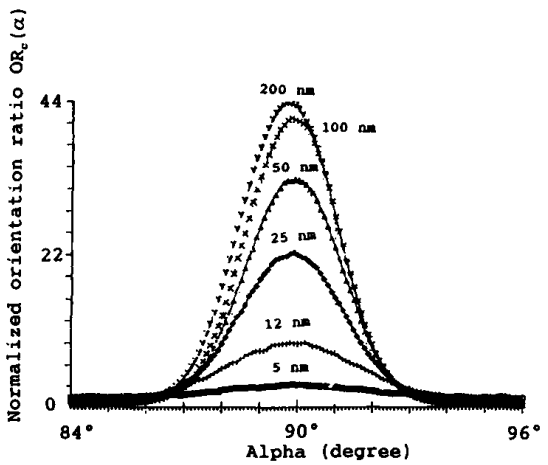

Fig. 10. The angular dependence of the orientation ratio $O R_{\mathrm{c}}(\alpha)$ of the crystallite for Series A films having different thicknesses.

from 5 to $200 \mathrm{~nm}$ for Series A films, the orientation ratio $O R_{c}$ (maximum of $O R_{c}(\alpha)$ ) monotonically increases from 3.5 to 44 . In the thinnest film of $5 \mathrm{~nm}$ the aspect ratio is close to 1 , and grains are equiaxed. However, even in this film, the orientation ratio reaches 3.5 , implying that (0002) texture is present, although the grains of the film do not exhibit a typical columnar shape.

Two important parameters are obtained from the angular dependence of the orientation ratio of the films: the peak value $O R_{c}$, reflecting the strength of (0002) texture and the $\Delta \alpha 50$ FWHM (full width-half maximum of the angular dependence of the $O R_{c}$ curve), reflecting the dis-

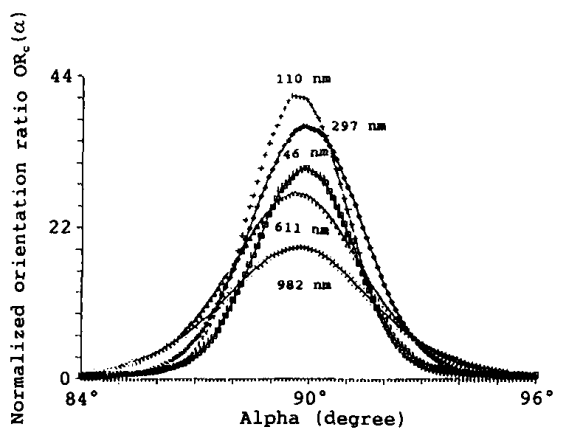

Fig. 11. The angular dependence of the orientation ratio $O R_{\mathrm{c}}(\alpha)$ of the crystallite for Series B films having different thicknesses.

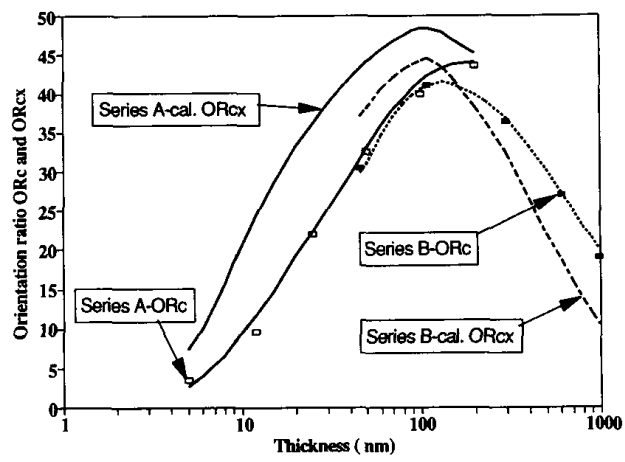

Fig. 12. The thickness dependence of the measured $O R_{c}$ values and the calculated $O R_{c x}$ values for Series A and $\mathrm{B}$ films.

persion of the $C$-axis. To obtain high quality perpendicular recording media, it is expected that a $\mathrm{Co}-\mathrm{Cr}$ film should have the highest possible $O R_{c}$ value and the lowest possible $\Delta \alpha 50$. The dependence of the peak orientation ratio $O R_{c}$ on the thickness is plotted in Fig. 12 for both Series A and B films. For Series A films (5-200 nm), $O R_{c}$ value increases constantly with increasing film thickness. For the Series B films, however, there is an optimum thickness where the maximum $O R_{c}$ value appears near 130 $\mathrm{nm}$. On the other hand, the dependence of $\Delta \alpha 50$ on the thickness in Fig. 13 shows that the optimum thickness, where the smallest $\Delta \alpha 50$ value is present, is at $50 \mathrm{~nm}$ and $80 \mathrm{~nm}$ for the Series A and B films. Thus the highest orientation ratio $O R_{c}$ and the smallest $\Delta \alpha 50$ value do not appear at the same thickness. A comparison of the magnitude of changes in the orientation ratio $O R_{c}$ and $\Delta \alpha 50$ shows that the relative changes of $O R_{c}$ are much larger than those of $\Delta \alpha 50$. For example, for Series A films, when the film thickness changes from 5 to $200 \mathrm{~nm}$, the relative change of $O R_{c}$ is as high as 12.6 , but the change rate of $\Delta \alpha 50$ is only 1.7 . It will be demonstrated in our next two papers [10], [12] on this subject that the orientation ratio $O R_{m}$ of the magnetization and the squareness ratio $R_{s}\left(M_{r}\right)$ $M_{s}$ ) for $\mathrm{Co}-\mathrm{Cr}$ films are mainly determined by the orientation ratio $O R_{c}$ of the crystallite, and only slightly influenced by $\Delta \alpha 50$. In view of this, it is recommended that 


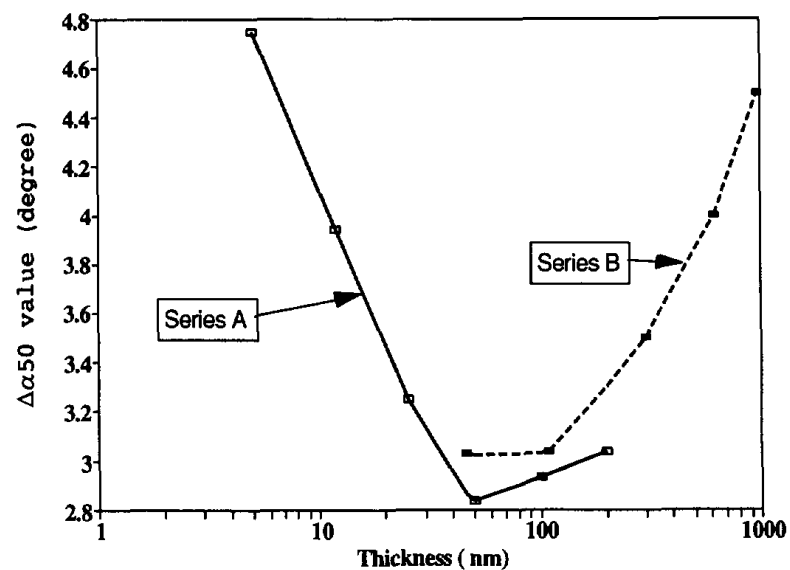

Fig. 13. The dependence of $\Delta \alpha 50$ on the thickness for Series A and B films.

$O R_{c}$ is used to represent the degree of crystallographic orientation in $\mathrm{Co}-\mathrm{Cr}$ films.

To explain why the orientation ratio of the crystallite decreases for films thicker than a critical value may involve the existence of stacking faults. These are considered to be related to internal stress. In general, it is believed [11], [17] that stacking faults may release some of the in-plane stress developed in films during grain growth. As well, based on observations made by other authors [6], [11], [16], columns themselves grow parallel to the film normal, but the $\mathrm{C}$-axis deviates from the direction of the grain growth in varying degrees. This will cause the $O R_{c}$ value to decrease in the thicker films.

The resulting orientation ratio $O R_{c}$ and $\Delta \alpha 50$ value are determined by a balance between grain growth and stacking fault formation. Looking at the measured $O R_{c}$ versus thickness curve for Series B films, if the film is thinner than the optimum value of $130 \mathrm{~nm}$, the improvement of crystalline alignment is the dominant factor resulting in an increase of the $O R_{c}$ value with increasing thickness. However, for a thickness greater than this optimum value, the number of stacking faults becomes dominant, and the dispersion of the hcp $C$-axes increases which decreases $O R_{c}$.

For an accurate analysis of experimental data, the influence of X-ray absorption (Mo-K $\alpha$ radiation) on the pole density must be considered. This absorption is a function of the film thickness and the attenuation ratio $\eta$ of the diffracted X-ray beam to the incident beam. This is given by [13]

$$
\eta=\frac{d I_{D T(\mathrm{at} x=T)}}{d I_{D O(\mathrm{at} \mathrm{x}=0)}}=\exp [-(2 \mu T / \sin \theta)],
$$

where $d I_{D O}$ and $d I_{D T}$ represent the intensities of the incident and diffracted beams. $\mu$ and $T$ are the linear absorption coefficient and thickness of the thin film, and $\theta$ is the incident angle of the X-ray beam. Obviously, the X-ray intensity diffracted from the top layer of the films contributes much more to the pole density than a beam diffracted from the bottom layer. In order to separate the net local orientation ratio $O R_{c x}$ of the crystallites for the $x$-th sublayer from the measured $O R_{c}$ value, the film is assumed to be divided into $x$ sublayers with different attenuation ratios $\eta_{x}$. The correlation between the normalized orientation ratio $O R_{c}(x)$ measured from the samples with changing thickness and the net local orientation ratio $O R_{c x}(x)$ corresponding to the $x$-th sublayer, corrected for the effect of X-ray absorption, can then be evaluated by the formulae [22]:

$$
O R_{c x}(x)=O R_{c}(x)+\frac{1-\exp (-\xi x)}{\xi} \frac{d O R_{c}(x)}{d x}
$$

With

$$
\xi=\frac{2 \mu}{\cos \alpha \sin \theta}
$$

where $\alpha$ and $\theta$ are the radial angles of the pole figure and the Bragg angle, and $x$ represents the distance of the $x$ th sublayer from the substrate along the film normal. The corrected local orientation ratio $O R_{c x}$ of the crystallite as a function of thickness for the Series A and B films is shown in Fig. 12. It can be seen from this figure that both the Series A and B films exhibit almost the same optimum thicknesses near $110 \mathrm{~nm}$.

Experimental data show that for Series A films, the $O R_{c x}$ value is always larger than the measured $O R_{c}$ value. In the case of Series B films, though the $O R_{c x}$ value is larger than the $O R_{c}$ value only for thicknesses less than $160 \mathrm{~nm}$. This difference in the pattern of the change in the $O R_{c x}$ and $O R_{c}$ values for Series $A$ and B films can be attributed to the effect of the different substrates. These results demonstrate that to obtain a high quality $\mathrm{Co}_{81} \mathrm{Cr}_{19}$ film with a high degree of crystallographic orientation, it is important to choose both the optimum film thickness and the proper substrate.

Finally, it is interesting to note that for Series B films both the highest orientation ratio $O R_{c \max }$ and the lowest strain $\epsilon_{\min }$ are found for films whose thicknesses are about $130 \mathrm{~nm}$. These conditions are very favourable for preparing high quality perpendicular recording media.

\section{Conclusions}

The following conclusions can be drawn from our experiments:

1) For the Series $\mathrm{B} \mathrm{Co}_{81} \mathrm{Cr}_{19}$ films, the diameter of columnar grains increases with increasing thickness, with rapid increases taking place in films thinner than $200 \mathrm{~nm}$. The aspect ratio vs. thickness curve exhibits a linear relation with film thicknesses up to $1000 \mathrm{~nm}$. As the thickness increases from 40 to 1000 $\mathrm{nm}$, the grain shapes gradually changes from equiaxial to conical shape.

2) In all tested films, the $(0002)$ planes are well aligned with the film surface. The texture in the $\alpha$-Co phase has a high degree of rotational symmetry around the film normal. 
3) Experimental data shows that for Series B films, the orientation ratio of the (0002) planes as function of the film thickness has a single peak near $130 \mathrm{~nm}$ where the orientation ratio $O R_{c}$ reaches its maximum value of 44. An analysis of the local orientation ratio $O R_{c x}$ of the $x$ th sublayers for Series $\mathrm{A}$ and B films shows the same optimum thickness (110 $\mathrm{nm})$, where $O R_{c x}$ values are as high as 48 and 45 . In our case, it was found that the orientation ratio $O R_{c}$ is the most sensitive measure of the degree of (0002) texture.

4) Both the orientation ratio $O R_{c}$ and the strain $\epsilon$ of the whole film depend strongly on the film thickness. The surface strain, however, is almost independent of the thickness.

5) The aspect ratios for films thinner than $46 \mathrm{~nm}$ is about 1 with no typical columnar structure. However, for these films the number of textured (0002) planes is still about 3-4 times stronger than in a random specimen.

\section{ACKNOWLEDGMENTS}

The authors acknowledge the financial support of the Natural Sciences and Engineering Research Council of Canada. We would like to thank Dr. W. Geerts and Dr. P. ten Berge, University of Twente, the Netherlands, for providing the samples and related experimental data of the magnetic properties. The authors are also obliged to Mr. S. Poplawski for doing the measurements on X-ray diffraction and texture. We are thankful to $\mathrm{Mr}$. A. Morawiec for calculating $O R_{c x}$ value.

\section{REFERENCES}

[1] J. G. Zhu and H. N. Bertram, IEEE Trans. Magn., vol. Mag-27, no. 4, pp. 3553-3562, 1991 .

[2] S. Onodera, K. Ouchi, Y. Nakamura and S. Iwasaki, J. of Magn. Society of Japan, vol. 15, no. 2, pp. 163-166, 1991.

[3] C. H. L. Goodman, Crystal Growth, Theory and Techniques, 1974.

[4] K. Reichelt, Vacuum, vol. 38, pp. 1083-1098, 1988.

[5] P. ten Berge, C. Lodder, S. Porthum, and T. Popma, J. Magn. Magn. Mater., vol. 113, pp. 36-46, 1992
[6] H. Cura and A. Lenhart, J. Magn. Magn. Mater., vol. 83, pp. 72, 1990.

[7] C. P. G. Schrauwen, J. P. C. Bernards, R. W. de Bie, G. J. P. van Engelen, H. H. Stel, V. Zieren, S. B. Luitjens, IEEE Trans. Magn., vol. Mag-24, no. 2, pp. 1901, 1988.

[8] M. Mirzamaani, C. V. Jahnes and M. A. Russak, IEEE Trans. Magn., vol. 28, no. 5, pp. 3090-3092, 1992.

[9] R. Hergt, H. Pfeiffer, and L. Fritzsch, Phys. Sat. Sol. (a) vol. 98, pp. $69-79,1986$.

[10] Li Cheng-Zhang, J. C. Lodder, and J. A. Szpunar, "The influence of the textural development on magnetic properties in $\mathrm{Co}_{81} \mathrm{Cr}_{19}$ films," J. Magn. Magn. Mat., vol. 131, pp. 427-439, 1994.

[11] B. G. Demczyk, J. Magn. Magn. Mater., vol. 102, pp. 238-246, 1991 .

[12] Li Cheng-Zhang, J. C. Lodder, and J. A. Szpunar, "The effect of the composition and the substrate on the texture evolution and magnetic properties in Co-Cr films," J. Magn. Magn. Mat. (submitted).

[13] B. D. Cullity, "Elements of X-ray diffraction," 2nd Edition, 1978

[14] R. S. Tebble and D. J. Craik, "Magnetic materials," pp. 68-73, 1969.

[15] J. C. Lodder, T. Wielinga, and J. Worst, Thin Solid Films, vol. 101, pp. $61,1983$.

[16] K. Hono, B. G. Demczyk, and D. E. Laughlin, Appl. Phys. Lett., vol. 55 , pp. $229,1989$.

[17] R. C. O'Handley and S-W. Sun, J. Magn. Magn. Mater., vol. 104107 , pp. 117-1720, 1992.

[18] Ch. Morawe, A. Stierle, N. Metoki, K. Brohl, and H. Zabel, J. Magn. Magn. Mater., vol. 102, pp. 223-232, 1991.

[19] B. G. Demczyk and J. O. Artman, J. Phys. D: Appl. Phys., vol. 24, pp. $1627-1632,1991$.

[20] W. J. M. A. Geerts, J. C. Lodder, and Th. J. A. Popma, J. Magn. Magn. Mater., vol. 104-107, pp. 971-972, 1992.

[21] T. Masuda, W. J. M. A. Geerts, and J. C. Lodder, J. Magn. Magn. Mater., vol. 95, pp. 123-132, 1991.

[22] A. Morawiec, "Pole figures of through-thickness inhomogeneous samples,"' internal report, McGill University, Montreal, Canada, 1992 .

Li Cheng-Zhang, biography not available at the time of publication.

J. C. Lodder, biography not available at the time of publication.

J. A. Szpunar, biography not available at the time of publication. 